\title{
OBESITY
}

\section{What's your fat-cell allowance?}

In the American sitcom Friends, Monica bakes mouth-watering cookies for her housemates, but - having been overweight as a teenager (pictured) - she is reluctant to eat them herself. Kirsty Spalding and colleagues now provide scientific evidence for why Monica has every reason to be cautious (K. L. Spalding et al. Nature doi:10.1038/nature06902; 2008).

Two factors contribute to an increase in fat mass: the number of fat cells and how much fat each of them stores (their volume). The authors studied the dynamics of fat-cell number in some 700 adults, both lean and obese, and combined their data with previous observations in children and adolescents.

A clear pattern emerged: irrespective of weight, the number of fat cells seems to rise steadily from birth to the early twenties, but remains constant thereafter. Moreover, in patients observed before and up to two years after surgical treatments that facilitate weight loss by reducing stomach size, no decrease in fat-cell numbers was detected - although their volume did drop.

So, are fat cells that are generated in early life doomed to remain with us till death us do part? In animal studies, this question can be addressed by labelling DNA nucleotides with radioactive isotopes such as ${ }^{14} \mathrm{C}$. Differentiated fat cells do not divide, and so radioisotopes, incorporated in their DNA in the last round of division before differentiation, remain there throughout the cells' life. The time of radiolabel incorporation, which is worked out from its half-life, is therefore the 'birth date' of these cells. But the potential toxicity of radioisotopes means that such studies cannot be performed in humans.

Spalding et al. cleverly thought of the next-best option. Atmospheric levels of ${ }^{14} \mathrm{C}$ have remained relatively constant for centuries, with the only major increase occurring between 1955 and 1963, when nuclear bombs were being tested above ground. A chain of reactions ensures that, at any given time, the radioisotope content of human DNA matches that of the atmosphere. The authors could thus follow fat-cell dynamics in individuals born around 1955-63.

As Spalding and colleagues' results show, fat cells have a high turnover: new cells are continually being born to replace their dead predecessors. The average age of a fat cell seems to be about 10 years in both lean and obese individuals, and the number of fat cells as a proportion of all cells remains constant in each weight group. But the total number of new fat cells was higher in obese subjects, suggesting that they are replenishing an existing larger pool.

So do the lean among us need to worry about our diet if we have fewer fat cells? Yes, we do: our fewer fat cells can still store large amounts of fat. Also, can obese people do anything about their weight? After all, they've already accumulated a

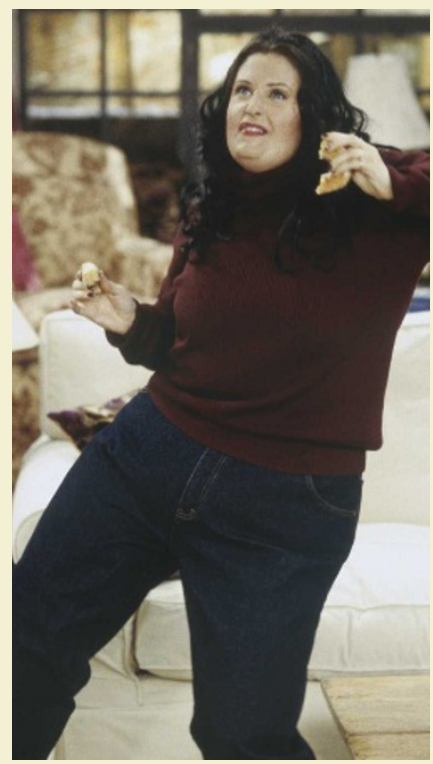

large pool of fat cells in childhood and adolescence? Again, the answer is yes. As Monica seems to have guessed, they can still reduce the volume, if not the number, of their fat cells. A further corollary of the paper is that researchers seeking drugs to cure obesity should consider targeting the mechanisms underlying fat-cell turnover.

Sadaf Shadan

\section{MOLECULAR BIOLOGY}

\section{An HIV secret uncovered}

\author{
Eddy Arnold and Stefan G. Sarafianos
}

\section{With two catalytic activities and many substrates, how does HIV's reverse transcriptase enzyme know what to do to which substrate? Zooming in on the enzyme's molecular interactions provides tantalizing clues.}

To replicate within their host cell, retroviruses such as HIV must make a double-stranded DNA copy of their single-stranded RNA genome. This elegant process, by which a viral enzyme - reverse transcriptase - synthesizes some 20,000 nucleotides, with the flow of genetic information moving in the opposite direction to normal (DNA to RNA), is nothing short of amazing. It also provides numerous opportunities for therapeutic intervention, as reverse transcriptase is the target of nearly half of the drugs approved for treating AIDS. But reverse transcription is not simple, for the enzyme comes into contact with diverse nucleic-acid substrates (DNA and/or RNA). On page 184 of this issue, Abbondanzieri et al. ${ }^{1}$ describe how this enzyme discriminates between substrates at intermediate steps of reverse transcription.

Reverse transcriptase has two distinct enzymatic activities: it is a DNA polymerase capable of copying either an RNA or a DNA template into a complementary DNA sequence; and it is an RNase $\mathrm{H}$, capable of degrading the RNA strand of an RNA-DNA duplex into small pieces once it has been used as a template for the first DNA strand (called the 'minus' strand; Fig. 1). But the enzyme overlooks some RNA segments, called polypurine tracts (PPTs), sparing them from degradation; these segments have an unusual sequence (and so structure), which prevents the enzyme's RNase $\mathrm{H}$ domain from cutting them ${ }^{2}$.

Like other DNA polymerase enzymes, reverse transcriptases initiate DNA synthesis using short nucleic-acid segments called primers. The primer used by HIV reverse transcriptase to generate the minus-strand DNA is a host-cell transfer RNA sequence. For the formation of the second (plus) DNA strand, which is complementary to the minus strand, the enzyme uses PPT segments that it has

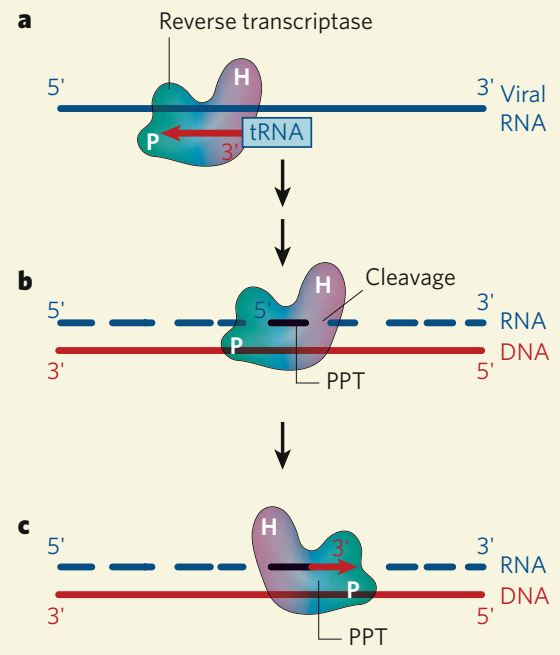

Figure 1 | Reverse transcription. Like other reverse transcriptase enzymes, that of HIV mediates transcription of the virus's RNA genome into double-stranded DNA. a, To form the first DNA strand (the minus strand) the enzyme uses a transfer RNA as a primer and interacts with the tRNA 3 ' end in a polymerase $(\mathrm{P})$ binding mode. b, As the complementary minus-DNA sequence is being synthesized, the enzyme cleaves the RNA template (but leaves the PPT sequences of the RNA intact) by binding to it in an RNase $\mathrm{H}$ (H) mode. c, Finally, to initiate the synthesis of the second (plus) DNA strand, the reverse transcriptase uses the PPT sequence as a primer, once again binding in the polymerase mode. 